\title{
Discovering dark matter
}

\section{Subir Sarkar*}

University of Oxford and NBI Copenhagen

E-mail: s.sarkar@physics.ox.ac.uk

Much effort has been devoted to the study of weak scale particles, e.g. supersymmeteric neutralinos, which have a relic abundance from thermal equilibrium in the early universe matching that of the dark matter. This does not however provide any connection to the comparable abundance of asymmetric baryons, which must have a non-thermal origin. 'Dark baryons' from a hidden sector with a similar asymmetry and mass of $\mathscr{O}(5) \mathrm{GeV}$ would naturally provide the dark matter. Lowthreshold direct detection experiments are required to find such particles, while monojet searches at colliders provide a complementary probe.

Frontiers of Fundamental Physics 14 - FFP14,

15-18 July 2014

Aix Marseille University (AMU) Saint-Charles Campus, Marseille

\footnotetext{
* Speaker.
} 